EVS26

Los Angeles, California, May 6-9, 2012

\title{
Building the Business Case for Telematics Based Diagnostics at Mahindra Reva
}

\author{
Kartik Gopal, R. Prakash \\ Mahindra Reva Electric Vehicles Pvt. Ltd., 122E, Bommasandra Industrial Area, Bangalore India, \\ gopal.kartik@mahindrareva.com, r.prakash@mahindrareva.com
}

\begin{abstract}
Mahindra Reva began a program of developing telematics capabilities in its electric vehicles around 4 years ago. The primary motivation for considering this technology was to enable remote diagnostics capabilities for our cars that are spread over 24 countries. This paper describes the evolution of telematics enabled capabilities at Mahindra Reva. This paper is divided into four broad sections: (1) we first present a technical overview of the telematics system; (2) we then briefly describe the categories of beneficiaries of telematics and the features and benefits to each category; (3) we then present the results from a survey of our existing battery electric vehicle owners on their perceived utility for such features; (4) based on these we develop a simple cost-benefit analysis to enable investment decisions in this technology and understand the payoffs from the investment.
\end{abstract}

Keywords: battery management system (BMS), controller, data acquisition, business model

\section{Introduction}

Telematics enables remote monitoring, control and data flows between vehicles and remote stations using wireless communication technologies. Enabling wireless communication between a vehicle and a remote station enables new modes of controllability and observability of the vehicle while also enabling a host of data-rich services to be offered in-car. The remote monitoring and control features are useful for offering emergency assistance in the event of vehicle break-down or accident, tracking and disabling of stolen vehicles, and remote diagnostics. The data flows enable connectivity to the web and to navigation systems, thus enabling new forms of value added services to be offered in-vehicle.
Telematics based features and services for passenger cars have evolved over the years, starting from 'high-stakes, rare-event' emergency response services offered by early models of GM OnStar system, to 'always-on' data-rich services being integrated into a host of cars today, an example being the Mercedes Benz mBrace system. The earliest models of GM's OnStar ${ }^{[1]}$, one of the earliest commercially launched telematics systems for personal vehicles, focused on emergency services and stolen vehicle tracking. The current offerings include turn-by-turn navigation and other services as an optional add-on. Other automakers are offering services ranging from live traffic updates, to data-rich $3 \mathrm{G}$ wireless data services including connectivity to the web (e.g. mBrace2 announced at CES in Janurary 2012 $2^{[2]}$ ) in their vehicles.

These new services come at a cost - most certainly to the vehicle manufacturer and possibly to the 
customer as well if she or he chooses to avail of such services. Costs can include the in-car equipment costs, the data-centre and call-centre setup costs, marketing costs and recurring operating expenses. The operating costs include administrative expenses and charges for wireless services to the car and the data and call centres.

There are some notable issues surrounding telematics costs for personal vehicles. First, it is clear that availing telematics services is a discretionary spend on the part of the customer else automakers would include telematics as standard fitment in cars and charge the customer for it. Automakers are still looking for the 'killer app' that could make telematics irresistible for consumers i.e. the set of telematics enabled features that will move customer preference for them from 'discretionary' to 'must-have'. This is further complicated by the fact that customers may chose to discontinue services after a certain period of usage. Lastly, telematics incurs recurring charges in the form of paying to telecommunication service providers for wireless connectivity services, the alternative being the automaker strikes a deal for supporting wireless services for a fixed period regardless of customer enrolment for availing telematics services, which adds cost and/or financial risk for automakers.

In spite of these factors, most major internal combustion engine (ICE) automakers are investing in these technologies and offering it in their cars. Presumably, there is a business case to be made that consumers are willing to discretionarily spend on such services. In fact, the services are increasingly becoming richer in data-bandwidth requirements and implementation complexity. It is also quite possible that automakers are embracing these technology to distinguish their cars in the market and increase sales, as is claimed in the case of the Ford SYNC ${ }^{\mathrm{TM}[3]}$ system.

Electric vehicle makers, especially makers of battery electric vehicles, are faced with a unique set of opportunities and challenges when compared to their ICE counterparts. On the one hand, electric vehicles, being significantly more 'electronics- rich' compared to conventional ICE vehicles, are inherently more amenable to data monitoring and control functions, making it somewhat easier to implement such features and functions. On the other hand, being a lowvolume segment by sales (certainly so when compared to ICE vehicle volumes), justifying the cost of introducing such systems may be challenging.

Is there a business case for makers of electric vehicles, and in particular automakers only producing battery electric vehicles (BEVs), to invest in introducing telematics services for their vehicles, and if so, how is it justified? In this paper, we examine the case for introduction of telematics at Mahindra Reva Electric Vehicles Pvt. Ltd. (MREV), a maker of BEVs based in Bangalore, India, where both authors are working.

We describe the telematics features being implemented for upcoming models from MREV. We also describe the results of a survey of existing MREV BEV owners to elicit their opinion about such services. We then describes the various factors that affect the price of offering such services and how these can be evaluated and justified in terms of benefits to customers and/or to OEMs We present a simple analysis for evaluating the potential benefits from telematics.

\section{Telematics System Description}

MREV has the longest history of continuous commercial BEV production, having produced around 4,400 cars to-date. The first of these cars was sold in India in 2001 and introduced in the UK in 2004. Since then, these cars have been sold in 24 different countries. The BEV's are sold as the Reva-i in India and other countries excluding the UK, where they are sold as the G-Wiz. An upcoming model, the NXR, is a new design with lithium-ion batteries and is larger in size and power.

MREV's telematics system comprises of on-board and off-board components that facilitate two way interchange of information and instructions between the car and the external world.

At the heart of the on-board intelligence is the "intelligent Energy Management System (iEMS)" which collects, collates, analyses, stores and communicates data.

An on-board communication gateway, the "Reva Telematics Gateway Unit (RTGU)" acts as the channel for the iEMS to communicate with the external world. Every Reva car is fitted with an RTGU, making it an integral part of the system. 
A web server located off-board acts as the 'central intelligence' collecting and collating information from the car.

A brief overview of the complete system is shown in Figure 1 below:

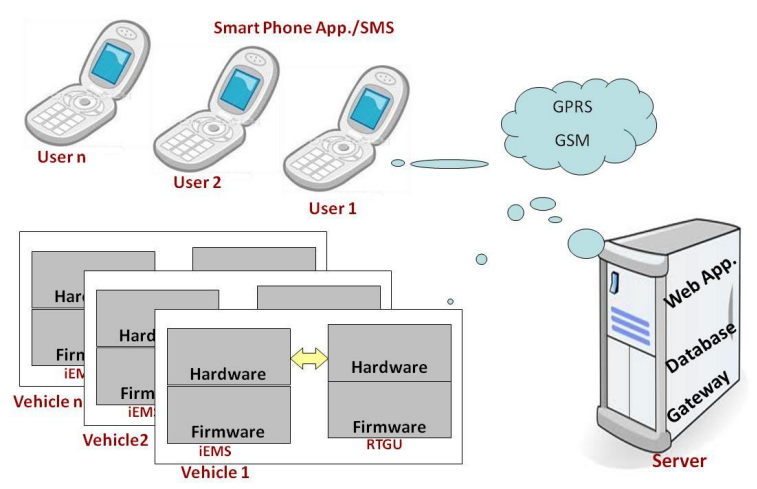

Figure 1: Reva Telematics system architecture

The server is capable of communicating with several vehicles at a given time. The telematics system also incorporates a user interface by means of smart phone applications.

The following subsections cover the functions performed by each of the subsystems of the telematics system

\subsection{The iEMS}

The iEMS incorporates several monitoring and management functions in the car spanning all energy transactions.

Some of the most important functions are in the areas of (see Figure 2 for a pictorial representation):

- Battery Management

- Drive monitoring

- Climate control

- User information

- Diagnostics

- Data management

The Battery management functions of the iEMS include:

- Administration of charge profile

- Thermal Management of the battery pack

- Continuous monitoring of parameters

- Cell balancing

- Calculation of SOC, $\mathrm{SOH}$

- Protections against abuse,
- Lifetime performance monitoring, diagnostics and user information.

All these functions are built in a flexible and modular fashion to facilitate quick adaptation to different battery string lengths and different battery chemistries

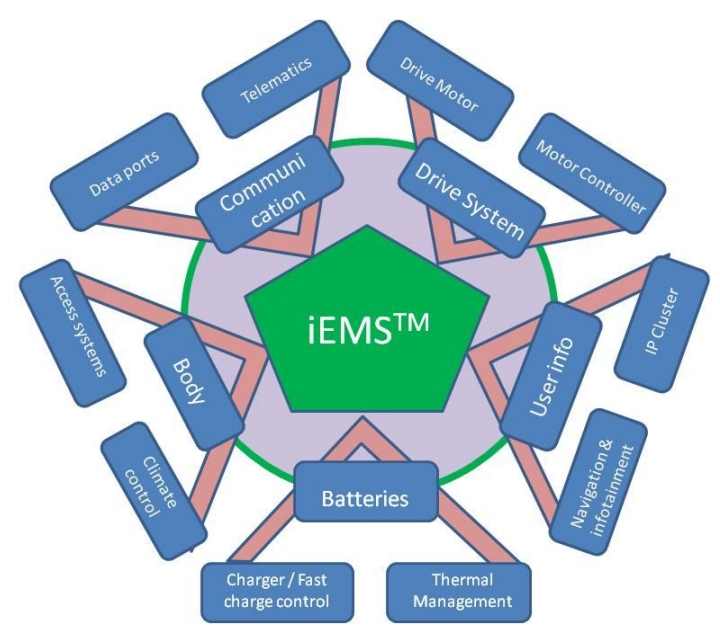

Figure 2: iEMS System Functions

The iEMS also keeps continuous tab of the health of the drive system by communicating with the motor/ controllers over a CAN bus link.

Other drive system functions built into the iEMS include:

- Drive energy monitoring,

- Regenerative energy monitoring, and,

- Supervisory controls to intervene during fault and 'impending fault' situations.

The iEMS also controls and monitors other major energy consumers like the climate control system from the point of view of

- Usage facilitation

- Energy monitoring and diagnostics.

Command structures in the iEMS allow car users to access the climate control through telematics. This allows users to preheat or precool the car when plugged in and conserve the battery energy for use during drive.

The energy management functions of the iEMS also extend to other energy consumers in the car and also cover other energy sources like solar panels. 
These 'real time' control functions of the iEMS are supplemented by a strong data management and analysis engine that incorporates the following among other important functions:

- On-board calculation of important performance parameters (Eg: Drive efficiency)

- On-board storage of historical data including battery lifetime behaviour.

- Collation of data from other 'intelligent subsystems'.

- On demand supply of all data through the gateway.

- On-board diagnostic algorithms to pinpoint any system issues.

The iEMS is thus built to be a 'single' point of contact for the external world to monitor car performance.

\subsection{The RTGU}

The RTGU is, as the name suggests a gateway for all the information exchange. Essentially a piece of 'embedded electronics' built around a GSM/ GPRS modem, the RTGU features a basic level of on-board intelligence required to ensure smooth data transactions.

The RTGU also incorporates mechanisms to -

- Recognise different classes of information and ensure proper routing

- Buffers to ensure information integrity and error detection/ handling.

The RTGU is built with a SIM card that uniquely identifies the car. The transactions through the RTGU cover:

- Alerts from iEMS to server

- Data requests from server to iEMS

- Data flow from iEMS to server and

- Commands from server to iEMS.

\subsection{The Server}

The server serves both as the repository of all data and also as the central intelligence in the telematics system. Some of the attributes of the server are:

- Ability to communicate with multiple cars at the same time

- Ability to communicate to multiple users (smart phone apps)

- Management of sessions, connections and user classes (multiple privilege levels)
Important functions built into the server include:

- Managing scheduled / periodic downloads of data from the entire population of vehicles.

- Accepting alerts from individual cars, prioritising them for action.

- Passing select alerts to user

- Automatic initiation of diagnostic sequence for select alerts.

- Initiating data downloads

- Initiating automatic self tests

- Analysis of stored data to understand

- Battery ageing patterns

- Lifetime behavior

- Usage and drive patterns

- Other subsystem behaviour

- Early detection of impending problems

- Charging management
○ Off-board charging / fast charging
- Charge pattern analysis
- Billing administration

Server applications also facilitate specific functions like

- User commands to the car

- Preheat, Precool, car access (i.e. door lock/unlock).

- Reserve Energy administration

- REVive ${ }^{\mathrm{TM}}$

\section{3 'Consumers' of Telematics Data}

The above 'telematics infrastructure' is designed to serve the needs of three classes of 'consumers of telematics data' - the 3 categories of customers as described below.

\subsection{The vehicle owner/driver}

We can broadly classify features that benefit the driver or owner of the vehicle into "range-anxiety alleviating features" and "utilitarian features".

\subsubsection{Range-anxiety alleviating features}

BEV's and their ecosystems have certain inherent disadvantages that telematics helps to overcome to various degrees. First, BEV's have a limited range and this requires the user to be sure she or he can make the journey before embarking on it. Secondly, public charging infrastructure is gradually being rolled-out and it will be some time before the same can become widespread. Users tend to feel apprehensive to use BEVs given these issues. 
Some BEV automakers offer a smartphone application along with their cars. The application enables customers to 'stay in touch' with their EVs by communicating with the car via telematics. Users can check the currently available range and battery charge at any point in time. Furthermore, they can plan their journeys and confirm whether the available charge in their cars is sufficient to make their intended trip. They can also locate nearby charging points and battery swap stations via the smartphone. These functions enable users to mitigate range anxiety. These features are not needed with ICE cars and with plug-in hybrids (PHEVs) but are essential with BEVs.

\subsubsection{Utilitarian features}

A second set of features, the so-called "utilitarian features", allow customers to interact with their vehicles in a manner that is unique to a BEV and PHEVs. An example of such functions is remote cabin temperature conditioning (cabin preheating or pre-cooling) and remote scheduling and control of vehicle charging. In addition, customers can receive reminders, alerts and notifications (for example, sending a reminder to plug-in) that can assist the customer or user adjust to the EV paradigm and make the EV usage experience more hassle-free.

A host of telematics based, customer-centric features that are common to EVs and ICE vehicles such as navigation and real-time traffic updates, can also be made available using the architecture described.

From the perspective of making an investment decision to support telematics, it may be noted that the inherent range anxiety issues make telematics services seem more necessary for BEVs than for PHEVs and ICE vehicles.

\subsection{The service and diagnostics personnel}

Telematics enables real-time performance data to be made available the automaker. Depending on the capabilities of the in-vehicle telematics system, it is even possible to communicate occurrence of faults and exceptions in the car and information relevant to debugging the root-cause of such faults. This singular features creates remote-diagnostics capabilities that can benefit the automaker and the customer through reduced service down-times, faster diagnosis of problems, and possibly even prognostics.

These telematics capabilities can also possibly help reduce the time lag in communicating problems observed in the field caused by defective manufacturing processes back to the factory floor.

\subsection{The research and development team}

Data aggregated from all of the automaker's vehicles on-road can help automakers understand vehicle and component performance under various usage and environmental conditions. With electric vehicles sporting newer battery technologies, reallife data on battery cycle-life and calendar-life under diverse usage and environmental conditions are relatively scarce. With data gathered from cars on-road using telematics, automakers can create and/or validate life-cycle models and models for predicting failures. It is entirely possible to have long term benefits in the form of being able to design cars better and faster.

\section{Survey of BEV drivers on Utility of Telematics Services}

MREV customers are early adopters of BEVs and they have experienced electric mobility without the benefit of telematics services. To understand customer preference for BEV centric telematics features as outlined in section 3.1, MREV carried out a survey of its existing customers. We had three goals for the survey:

a. Estimate the degree of interest in EV centric telematics based smartphone features to address range-anxiety;

b. Estimate the degree of interest in EV centric utilitarian features using smartphones;

c. Estimate their willingness to pay for such services.

MREV gathered data for this research by means of survey questionnaires administered over the internet and also from face-to-face interviews with customers. The results can be summarized as follows:

a. Over $70 \%$ of respondents expressed a strong interest for each range-anxiety alleviating feature such as knowing available charge in the car, finding the nearest charging station, planning a trip, time remaining for full-charge, etc. 
b. Less than $30 \%$ of respondents expressed any interest in utilitarian features such as remote charging, cabin conditioning, remote door lock/unlock.

c. However, at least $65 \%$ of respondents sought alerts and notifications of things going wrong (for example, getting a notification when the car is plugged in for charging but it not getting charged).

d. When asked about their willingness to pay for such features, 35\% said they should be offered free of cost while 39\% indicated they will decide after a trial.

We draw two conclusions from the above results: First, the results in points (a) - (c) seem to be a classic instance of the Freudian Pleasure Principle $^{[4]}$ at work - we wish to maximize pleasure while minimizing pain. The EV centric features consist of some that decidedly "reduce pain", namely features that reduce range anxiety and alerts and notifications that inform us that something went wrong with our car. A significant majority of respondents seek access to such features.

The utilitarian functions are not sought after by a majority of respondents - a result which seems to indicate that while these features are not about avoiding pain of any sort, they are also not adding any significant avenues for gaining 'pleasure'.

The above interpretation is corroborated by the responses to our queries on what motivates them to consider these features, if at all. Over $65 \%$ of respondents said they are interested in these features primarily for the 'anxiety-avoiding' benefits, while $\sim 38 \%$ expressed an interest in these features because they were 'cool'. It may be noted that the fraction of respondents who expressed an interest in utilitarian features was also similar $(\sim 35 \%)$. [Note: respondents were allowed to choose more than one reason, hence the tally is $>100 \%$ ]. Our responses did not record whether those who found utilitarian features desirable were also those who found them to be 'cool'. So it is not possible to draw any correlation between the two.

We can draw another conclusion from point (d) above. $74 \%$ of respondents were either unwilling or hesitant to pay for these features. We may infer that in the case of BEVs, customers expect $B E V$ automakers to make available any and all means to address range anxiety as a standard offering with the car without being charged for such features. One may stretch this further to admit the possibility that customers demand these features as a price automakers have to pay to enable customers to adopt BEVs, given the limitations of BEVs when compared to ICE or PHEV vehicles.

\section{Building the business case}

The voice of the customer clearly calls for telematics based features to be made a default 'component' of the BEV driving experience. This in itself could be considered as a sufficient reason for BEV automakers to include these features in their vehicles in order to gain widespread customer acceptance of BEVs and justify the increased cost.

We also examined whether and how telematics may reduce costs related to diagnostics and servicing of vehicles. We consider automakers such as MREV who only make BEVs. Such OEMs will face a unique challenge: they would need to sell in geographically dispersed areas to gain volume, but will find it costly to setup service centres in every region. Making BEVs at an affordable cost is challenging enough, building a geographically diverse service and support network adds significant expense.

In such contexts, telematics can play a vital role in reducing service costs by enabling remote diagnostics. We model this as follows:

In the scenario where telematics enabled remote diagnostics is not available, let us denote -

$T_{0}$ - the date and time instant at which a component failure occurs in the BEV;

$T_{1}$ - the date and time instant when symptoms manifest and customer becomes aware of a problem;

$T_{2}$ - the date and time instant when brings vehicle to the dealer or service centre;

$T_{3}$ - the date and time instant for dealer to have diagnosed problem;

$T_{5}$ - the date and time instant for dealer to carry out replacement/repairs;

In most cases, it can be seen that:

1. $\left(T_{2}-T_{0}\right)>\left(T_{3}-T_{2}\right)$.

2. The symptoms at $T_{1}$ may include issues such as the car not moving or stopping while driving - "catastrophic" scenarios that no customer ever wishes to face (pain point to be avoided). 
With telematics enabled diagnostics, the customer promise that an automaker wishes to strive for is -

1. $\left(T_{3}-T_{0}\right) \sim 0$

2. "Catastrophic" scenarios do not occur or help is made available in a timely manner.

In other words, the customer can be informed of the occurrence of a problem before she or he notices symptoms; furthermore, problem resolution time is reduced once the customer brings the vehicle in to a service centre. In many situations a service engineer can come over to the customer's location and fix the problem in-situ, or even better, a fix may be downloaded into the car 'over-the-air', thus saving the customer any need for a visit to the service centre and enabling the company to have fewer service centres to service vehicles in a given region. This benefit can have the highest cost impact to the company and potentially justify the cost of telematics. This benefit is predicated on the car being designed for ease-of-maintenance and serviceability where most parts can be accessed without having to mount the car on mechanical jigs.

We represent this scenario as follows:

Let,

Number of cars in a region $-\mathrm{N}$;

Number of cars served per service station $-\mathrm{N}_{\mathrm{cs}}$; Annual cost of a single service station (we amortize setup and operations cost to an annualized number) $=\mathrm{C}_{\mathrm{s}}$;

Then,

Number of service stations required in the region $=\mathrm{N}_{\mathrm{s}}=\mathrm{N} / \mathrm{N}_{\mathrm{cs}}$;

Let,

Annual cost of telematics per car (development, setup and operations cost annualized per car) $\mathrm{C}_{\mathrm{t}}$;

Number failures that require service personnel attention per car per year $-R_{c}$;

Percentage of $R_{c}$ that does not require service station infrastructure - Fs;

Then,

Given that $\mathrm{N}_{\mathrm{s}}$ service stations are required to service $(\mathrm{Rc} * \mathrm{~N})$ failures per year in the region without the help of telematics, (i.e. total number of car problems dealt with in the region by all $\mathrm{N}_{\mathrm{s}}$ service centres in the region), the number of service stations required when aided by telematics is reduced to:

$\mathrm{N}_{\mathrm{t}}=\mathrm{N}_{\mathrm{s}} * \mathrm{~F}_{\mathrm{s}}$

Cost savings from reducing number of service stations is -

$\mathrm{C}_{\mathrm{ss}}=\left(\mathrm{N}_{\mathrm{s}}-\mathrm{N}_{\mathrm{t}}\right) * \mathrm{C}_{\mathrm{s}}$

This leads to the conclusion that telematics cost is justified if:

$\mathrm{C}_{\mathrm{ss}}>\left(\mathrm{N} * \mathrm{C}_{\mathrm{t}}\right)$

Costs for service personnel to make in-situ visits and repair can be included into the annual telematics cost.

In addition to enabling the possibility of reducing service centre infrastructure, telematics enables the following relevant capabilities as well:

1. Reduces the time taken for failure related information to be communicated between dealer to service engineer to $R \& D$ especially for new models. With telematics, R\&D can get directed notifications on specific error conditions.

2. Over-the-air (OTA) customization of drives: electric vehicles are largely 'software driven'. It might be possible to fix certain failures through software code updates. With appropriate security mechanisms, this code update could be carried out 'over-the-air' without the car needed a visit to a service centre.

Given that BEVs will have a much slower paced uptake compared to traditional vehicles and that in order to create volumes, automakers will need to sell smaller volumes in geographically spread out regions or countries, it appears that opting for telematics based solutions can be a useful aid for $\mathrm{BEV}$ companies to reduce maintenance overheads both for themselves as well as for their customers.

\section{Summary}

This paper describes the telematics architecture implemented in Mahindra Reva's BEVs. It also presents insights from customers on their perceived need for various features possible via telematics in conjunction with battery electric vehicles. The paper also proposes an analytical approach to evaluating costs and benefits from a 
service perspective that accrues with a telematics system.

\section{References}

[1] Online http://www.onstar.com accessed on 2012Mar-4

[2] Online http://www.mbusa.com/mercedes/mbrace2 accessed on 2012-Mar-4.

[3] Online http://media.ford.com/article display.cfm? article $i d=27442$ accessed on 2012-Mar-4.

[4] Online http://www.bartleby.com/276/ "Beyond the Pleasure Principle" Sigmund Freud, accessed on 2012-Mar-4.

[5] EVS26, http://www.EVS26.org, accessed on 2012-01-06

\section{Authors}

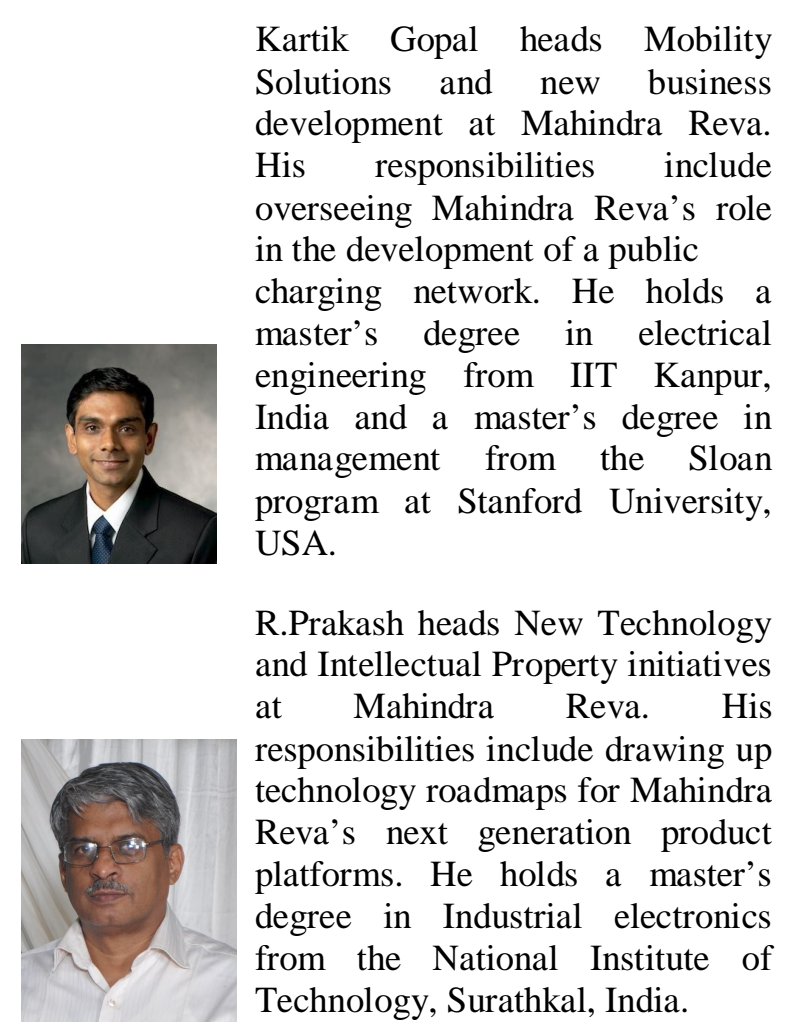

